\title{
Fachbeitrag
}

\author{
Achim Bonte
}

\section{Was ist eine Bibliothek? Physische Bibliotheken im digitalen Zeitalter}

Zusammenfassung: Während die landläufigen Vorstellungen von „Bibliothek“ bislang beunruhigend statisch bleiben, erzwingen die fortschreitende Digitalisierung von Medien und die Mobilisierung des Mediengebrauchs längst neue Bedeutungsinhalte. In Fachkreisen entwickelt sich die Idee service- und erlebnisorientierter Lernräume anstelle des klassischen Medienspeichers zu einem gängigen Leitbild. Doch wie aussichtsreich ist diese neue Schwerpunktbildung? Und wie wird dieser Anspruch tatsächlich erfüllt und nachhaltig gesichert? Ausgehend von den Umbrüchen in der gesamten Informations- und Medienbranche beschreibt der Beitrag wesentliche Rahmenbedingungen für das Innovationsmanagement in Bibliotheken und die Schlussfolgerungen, die daraus zu ziehen sind. Anschließend werden einige konkrete Entwicklungschancen für physische Bibliotheksorte vorgestellt.

\section{What is a library? Physical Libraries in the Digital Age}

\begin{abstract}
Up to now, the general image of a "library" has remained alarmingly unchanged. But the advancing digitization of media and the mobilization of media use require new meaning and content. Among experts the idea of service- and experience-oriented learning spaces evolves as a common concept, replacing the classical media storehouses. But how promising is this new concept? And how is this claim met and sustainably ensured? Based on changes in the information and media sector, the article describes the general framework for innovation management in libraries and the related conclusions. Subsequently, some concrete development opportunities for physical libraries are presented.
\end{abstract}

Schlüsselwörter: Bibliotheksbegriff; Bibliotheksentwicklung; Veränderungsmanagement

Keywords: Library Concept; Library Development; Change Management

DOI 10.1515/abitech-2015-0019
Eine Bibliothek ist eine Bibliothek ist eine Bibliothek. Obgleich ihr traditionelles Leistungsangebot zunehmend erschüttert wird, erweist sich die traditionelle Semantik von „Bibliothek“ bislang als äußerst stabil. „Eine Bibliothek oder Bücherei ist eine Dienstleistungseinrichtung, die ihren Benutzern Medien zur Verfügung stellt“, lautet zum Beispiel der erste Satz des aktuellen Wikipedia-Artikels. Und weiter unten: „Zentrale Dienstleistung der Bibliotheken ist die Ausleihe von Medien." ${ }^{1}$ Typischerweise stellt der übliche Bibliotheksbegriff bevorzugt auf Aspekte der „Sammlung“ und des „Medienspeichers“ ab, zudem noch meist fokussiert auf Printmedien und mit Berufsvorstellungen nach den Klischees des „Hollywood Librarian“2. Die Risiken dieser hohen Beständigkeit des tradierten Bibliotheksbegriffs und Berufsbilds liegen auf der Hand. Wenn Bibliotheken weiterhin hauptsächlich als Orte der Bücher und der Medienausleihe interpretiert werden, besteht angesichts der raschen Entwicklung der digitalen Informations- und Wissensgesellschaft eine wachsende Gefahr für deren Legitimität und Ressourcenakquise. Wie real dieses Risiko ist, lässt sich etwa daran ablesen, dass der Beruf des Bibliothekars nach Daten des US-Arbeitsministeriums bereits unter die „Five dead-end jobs“ fällt: „Librarians probably play a huge part in your childhood memories, but [...] it's likely that 'memory' could be the only role left for librarians to play." 3

Entsprechend bemühen sich Bibliotheksfachleute um ein zeitgemäßes und vielfältigeres Bibliotheksbild bis hin zu einer ,stark ausgeprägten, individuellen Profilbildung““4,

1 Bibliothek. In: Wikipedia, http://de.wikipedia.org/wiki/Bibliothek (3.3.2015).

2 The Hollywood Librarian: A Look at Librarians Through Film, 2007. Regie: Ann M. Seidl. Ein zum Teil dokumentarischer, zum Teil fiktionaler Film, der anhand zahlreicher Interviews mit Bibliothekarinnen und Bibliothekaren sowie der Darstellung von Bibliotheken in Filmen das Image von Bibliotheken ergründen will.

3 Duchon, Andrea: Five dead-end jobs, and how to escape them, http://www.imfjobtraining.org/education.yahoo.net/articles/ five_dead_end_jobs.html (3.3.2015).

4 Eigenbrodt, Olaf: Ist eine klare Definition von Bibliothek noch möglich? In: Buch und Bibliothek 65,2 (2013), S. 110-113, S. 113. 
jedoch wird derlei durch die regelmäßige Wiederkehr von eingefahrenen Denkmustern auch in der Fachöffentlichkeit allzu oft durchkreuzt: Zum Beispiel dadurch, dass man Führungen zu gern in den Schatzkammern beginnt, Veranstaltungshinweisen Bilder von prächtigen Lesesälen oder Regalreihen beigibt oder auf der Website des Nürnberger Bibliothekartages 2015, der wichtigsten Fortbildungsveranstaltung der Branche, alle Interessierten unbedacht mit einem romantischen Foto des Albrecht-Dürer-Hauses begrüßt. In der konkreten bibliothekarischen Bau- und Aufgabenplanung ergibt sich nicht selten der gleiche Eindruck. „Die Reformprozesse sind noch immer von einer Methodik geleitet, die auf umfangreichen Printbeständen aufsetzt“, bilanzieren Konstanze Söllner und Wilfried Sühl-Strohmenger im 2014 veröffentlichten „Handbuch Hochschulbibliothekssysteme“.5 „Wenn in Europa über Bibliotheksbau gesprochen wird“, so der Bibliotheksbauexperte Olaf Eigenbrodt im selben Jahr, „dann herrscht eine Zentralperspektive vor, deren Fluchtpunkt immer die Büchersammlung ist." ${ }^{6}$ Ebenfalls dazu passend erscheint der steinige Weg $\mathrm{zu}$ einem modernisierten, offeneren Berufsbild und vielleicht sogar der beharrliche Versuch, Bibliotheken mit der Parole „Bibliotheken als Pflichtaufgabe“ eher als gleichsam gefährdete Art unter gesetzlichen Schutz zu stellen, anstatt sie entschlossen von innen heraus mit neuem Bedeutungsinhalt in die Mitte auch künftiger Gesellschaften zu rücken. Zusammenfassend bleibt der Eindruck bestehen, dass die immensen Gefährdungen der nächsten Jahre oft weiter dramatisch unterschätzt oder leichtfertig verdrängt werden und die Geschwindigkeit der Entscheidungs- und Entwicklungsprozesse längst nicht der Veränderungsdynamik der Informationslandschaft entspricht. Als Lackmustest darf der Personaleinsatz in Bibliotheken gelten: Für die Papierbibliothek werden bisher fast ausnahmslos noch immer zu viel, für neue Aufgaben der digitalen Informationsinfrastruktur dagegen viel $\mathrm{zu}$ wenig Ressourcen aufgewendet. ${ }^{7}$

5 Söllner, Konstanze; Sühl-Strohmenger, Wilfried: Hochschulbibliothekssysteme 2020. Thesen und Perspektiven. In: Dies. (Hrsg.) Handbuch Hochschulbibliothekssysteme, Berlin 2014, S.563-566, S. 563.

6 Eigenbrodt, Olaf: Veränderte Kontexte und Funktionen. Ansätze einer neuen Typologie für Wissensräume. In: Eigenbrodt, Olaf; Stang, Richard (Hrsg.): Formierungen von Wissensräumen, Berlin 2014, S. 22-36, S. 23.

7 Vgl. Bonte, Achim: Wissenschaftliche Bibliotheken der nächsten Generation. Sind die Institutionen und ihre Mitarbeiter für die Zukunft gerüstet? In: Zeitschrift für Bibliothekswesen und Bibliographie 61,4/5 (2014), S. 239-242.

\section{Herausforderung Internet}

Jenseits von Begriffs- und Berufsbilddebatten sprechen die Tatsachen, vollzieht sich die Digitale Revolution mit zunehmend absehbaren, für Bibliotheken problematischen Ergebnissen. Das Internet ist bereits heute die maßgebliche Infrastruktur für die Recherche, Verteilung und Nutzung von Information und Wissen. Der Mobilisierung der Medien (mittels retrospektiver Digitalisierung bzw. Born-digital-Publikationen) folgt die Mobilisierung des Mediengebrauchs. Die Vision des mobilen Internets, digitale Inhalte und Dienste zu jeder Zeit, an jeder Stelle situationsangepasst und personalisiert nutzen zu können, ist bei zu vielen Bibliotheken noch nicht erkennbar angekommen, mit gut 33 Millionen verkauften Smartphones und Tablets im Deutschland im Jahr 2014 aber trotzdem eine Realität. ${ }^{8}$ Zur Revolution gehört inzwischen auch der Abstieg der Webseite als eines zentralen digitalen Ortes. Entsprechend sind Bibliotheksinhalte gewiss nicht mehr nur auf der eigenen Domain, sondern auch auf den Touchpoints von Google, YouTube, Twitter, Pinterest, Whatsapp \& Co. anzubieten. ${ }^{9}$ Zugunsten eines kontinuierlichen digitalen Stroms ,medial entgrenzter Inhalte“ lösen sich zunehmend sogar die tradierten Publikationseinheiten auf, womit ordnende Hilfskonstruktionen aus der Papierbibliothek, die Kategorien von E-Book und E-Journal, endgültig obsolet werden. ${ }^{10}$ Zugleich gewinnt die Forschung erweiterte Erkenntnischancen und grundlegend veränderte Methoden, wofür die wachsende Zahl von Digital-Science-Zentren und -Tagungen auch für die lange eher reservierten Geisteswissenschaften Zeugnis ablegt, und woraus Bibliotheken als die angestammten Labore der Geisteswissenschaften mit Blick auf so wichtige Aufgaben wie die Standardisierung und Akkreditierung von Daten, Formaten und Diensten ebenfalls dringend Schlüsse ziehen sollten.

Die gravierenden Folgen der Digitalisierung für die gesamte Informations- und Medienbranche lassen sich im privatwirtschaftlichen Sektor mühelos ablesen. Im Zeitungsangebot gingen in den letzten Jahren einst so bedeutende Blätter wie die Frankfurter Rundschau, der Rheinische Merkur oder die Westfälische Rundschau ver-

8 GfK im Auftrag des BITKOM, EITO (2014), http://www.bitkom. org/files/documents/BITKOM-Praesentation_CE_Pressegespraech_ 03_09_2014_02.pdf (3.3.2015).

9 Vgl. Eck, Klaus; Eichmeier, Doris: Die Content-Revolution im Unternehmen. Neue Perspektiven durch Content-Marketing und -Strategie, Freiburg, München 2014.

10 Vgl. Ceynowa, Klaus: Der Text ist tot. Es lebe das Wissen. In: Hohe Luft 1 (2014), S. 53-57. 
loren. Im Bereich der Publikumszeitschriften sind ganze Themengruppen ins Internet gewechselt. Die hektischen kompensatorischen Neugründungen haben geringe Auflagen, sehr spezielle Zielgruppen und häufig eine kurze Erscheinungsdauer. Im Börsenverein des Buchhandels ist die Mitgliederentwicklung stark rückläufig. Bei den Videotheken werben Video-on-Demand-Dienste unverhohlen mit der Losung „Sparen Sie sich den Gang zur Videothek“. Im erbitterten Kampf um das wirtschaftliche Überleben und neue Marktchancen verschwimmt die vertraute Ordnung der Angebotssegmente und bieten Qualitätszeitungen zum Beispiel inzwischen auch Rechtsberatung und professionelle Faktenrecherchen oder Buchhandlungen Spiele und Geschenkartikel an. In einer großen Buchhandelskette belegten diese Sortimente schon vor einigen Jahren rund 30 Prozent der Ladenflächen. Während die meisten Buchhandlungen diversifizieren, versuchen andere die Spezialisierung. Die „Krimibuchhandlung“, der „Frauenbuchladen“ oder die "Comicothek" wollen sich jeweils durch besondere Angebotstiefe, passendes Rahmenprogramm und überlegenes Fachwissen eine auskömmliche Marktnische sichern. Beiden Strategien gemeinsam ist das Bewusstsein der Notwendigkeit aktiven Marketings in einem strengen Wettbewerbsumfeld, des unausweichlichen Zwangs, laufend Produkte und Konditionen zu bieten, die durch genügend spezifische Merkmale und ausreichend akzeptierte Qualität auch tatsächlich konkurrenzfähig sind.
Bibliotheken sind als öffentliche Einrichtungen nicht unmittelbar den Marktzwängen ausgesetzt, folgen aber gleichwohl dem Prinzip von Angebot und Nachfrage. Wenn sie die Herausforderungen des Internetzeitalters längerfristig bestehen wollen, benötigen sie daher ebenfalls stets genügend Produkte, die ihnen anhaltend $\mathrm{Zu}$ stimmung sichern. Eine konsequentere marktwirtschaftliche Betrachtung der Diensteportfolios von Bibliotheken würde vermutlich zu folgenden Ergebnissen führen: Die Listen der Leistungsangebote würden in der Regel gestrafft, deren durchschnittliche Qualität verbessert und die echte Bereitschaft zur Zusammenarbeit unter den Häusern vermehrt. Mit dem Bemühen, jeweils vor Ort Bibliotheksprofile zu entwickeln, die sowohl möglichst gut zu gesetzlichem Auftrag und Betriebsgröße passen als auch möglichst viele Alleinstellungsmerkmale im Wettbewerb bieten, dürfte zugleich die Bandbreite dessen, was „Bibliothek“ kennzeichnen und bedeuten kann, signifikant zunehmen.

Wenn sich Bibliothekare vor den Härten des Marktes bisher vergleichsweise sicher wähnen, hat dies neben der schützenden öffentlich-rechtlichen Trägerschaft und der besonders langen historischen Tradition der Bibliothek als Kultur- und Wissenschaftsinstitution weitere Ursachen. Erstens befindet sich die Benutzung der Papierbibliothek zwar irreversibel auf dem Rückzug, erfüllt bislang und auch in der näheren Zukunft aber noch immer eine wichtige Teilfunktion. Diese ist umso weniger

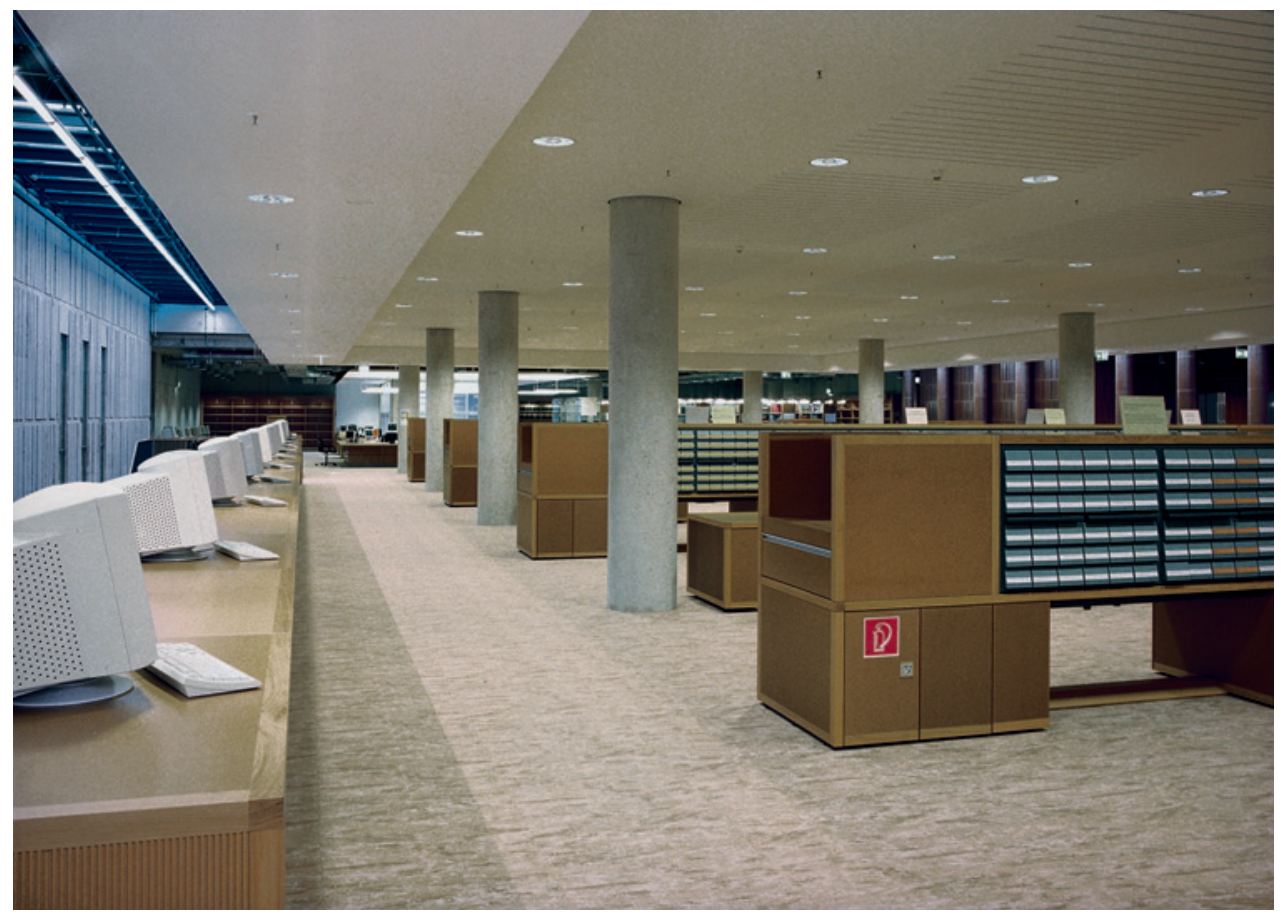

Abb. 1: Eingangsbereich ... der Zentralbibliothek 2002 ... (Foto: Henrik Ahlers, SLUB Dresden) 


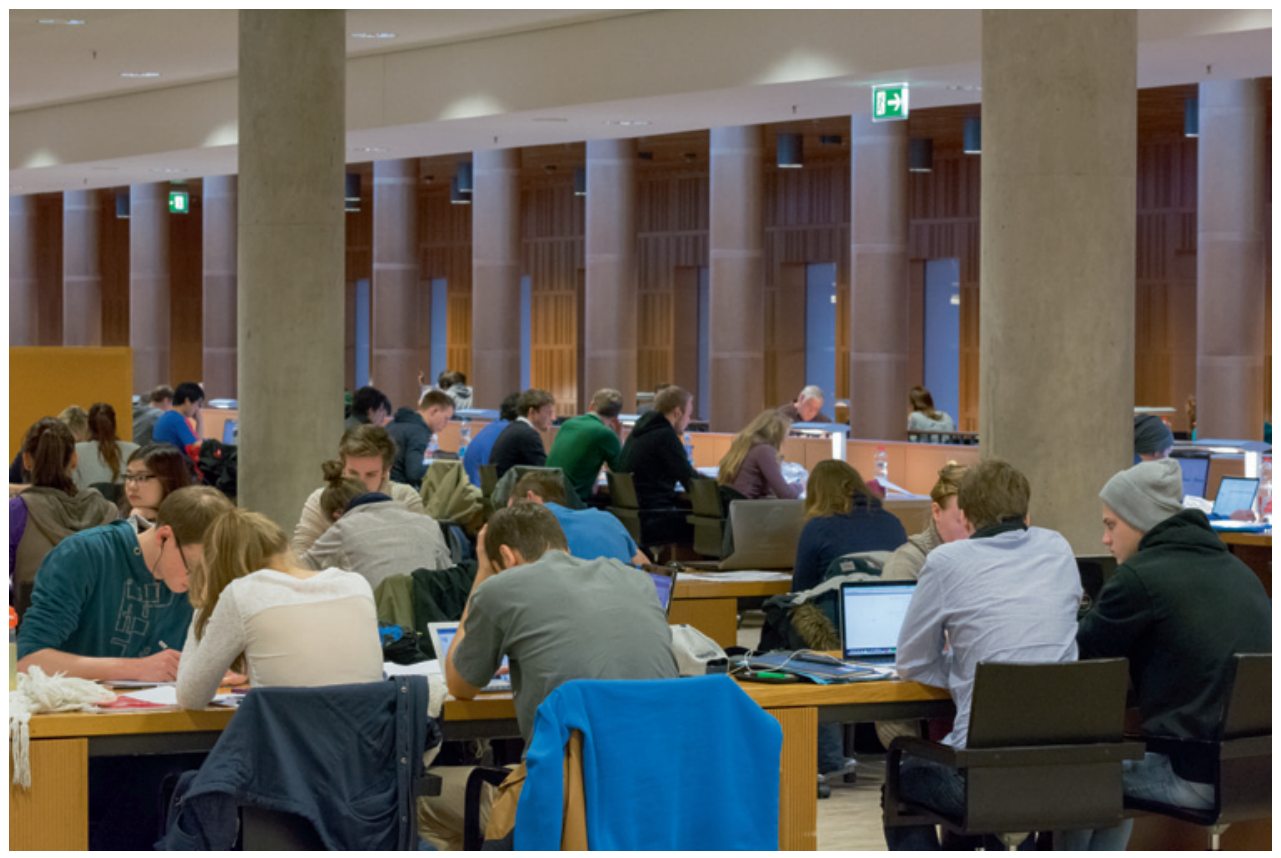

Abb. 2: ... und 2015 (Foto: Henrik Ahlers, SLUB Dresden)

diskreditiert, als sie ja - wie eingangs gezeigt - dem gültigen Bibliotheksbild der breiten Öffentlichkeit entspricht. Zweitens wird die rückläufige Nachfrage nach konventionellen Medien durch den Zugriff auf lizenzierte digitale Medien gegenwärtig oft überkompensiert. Ob das angesichts von Retrodigitalisierung, Open Access und Micro-Publishing nicht lediglich ein technisches Übergangsphänomen bleibt, steht dahin, ist ob dieser Unentschiedenheit aber ebenfalls aktuell noch nicht handlungstreibend. Drittens scheint mit der Propagierung der Bibliothek als lebendigem Kommunikations- und Begegnungszentrum bereits ein zuverlässiger Ausgleich gefunden, um die Institution auch langfristig abzusichern. Speziell aus dieser sozialen Funktion der Bibliothek bezieht die Branche derzeit einen gewichtigen Teil ihrer $\mathrm{Zu}$ kunftserwartung.

\section{Der attraktive Lernraum als neues Paradigma}

Hinsichtlich der Gestaltung von Bibliotheken als architektonisch anspruchsvolle, einladend gestaltete und komfortable Arbeitsorte mit vornehmlich kommunikativer Bedeutung ist in den letzten zwanzig Jahren in der Tat manches geschehen. Doch wie aussichtsreich ist die Strategie? Wie wird der Anspruch im Einzelnen gefüllt und nachhaltig gesichert? Welche neue Konkurrenzsituation ergibt sich daraus und worin liegt die bibliothekari- sche Spezifik gegenüber vergleichbaren Angeboten anderer öffentlich-rechtlicher Einrichtungen und der Privatwirtschaft? Auf eine Formel gebracht, gilt als verbreitetes Paradigma, die Kompetenzen von Amazon, Apple, Starbucks und eines Coworking-Space zu vereinen und in der konkreten Produktbildung derlei erfolgreichen Vorbildern nachzustreben. Amazon steht für die zügige, kundengesteuerte Content-Vermittlung rund um die Uhr, Apple für attraktive Geräte und intuitive Benutzeroberflächen, Starbucks für bequeme Sitzmöbel und zuverlässige kulinarische Versorgung, Coworking für die Chance auf stimulierende Begegnung und Wissensaustausch. Abgesehen davon, dass man sich mit einem solchen Profil jeweils am Qualitätsniveau der genannten Unternehmen messen lassen müsste, entsteht durch die starke Entkopplung der Funktionen von den klassischen Beständen und bestandsbezogenen Vermittungsleistungen ein Leistungskatalog, der keineswegs zwingend an die Institution „Bibliothek“ gebunden bleibt. Entsprechend kann zum Beispiel auch eine Institution wie das CoworkingSpace „betahaus“ in Berlin-Kreuzberg ein Angebot machen, das sich „between a Viennese coffee house, a library, a home office, and a school campus" ansiedelt; ${ }^{11}$ und was geschieht, wenn sich vergleichbar konkurrierende Konzepte direkt an Hochschulen etablieren? Vorerst mehr als Gedankenspiel entwarfen zum Beispiel Architekten an der Technischen Universität Dresden eine

11 Vgl. http://www.betahaus.com/berlin (3.3.2015). 


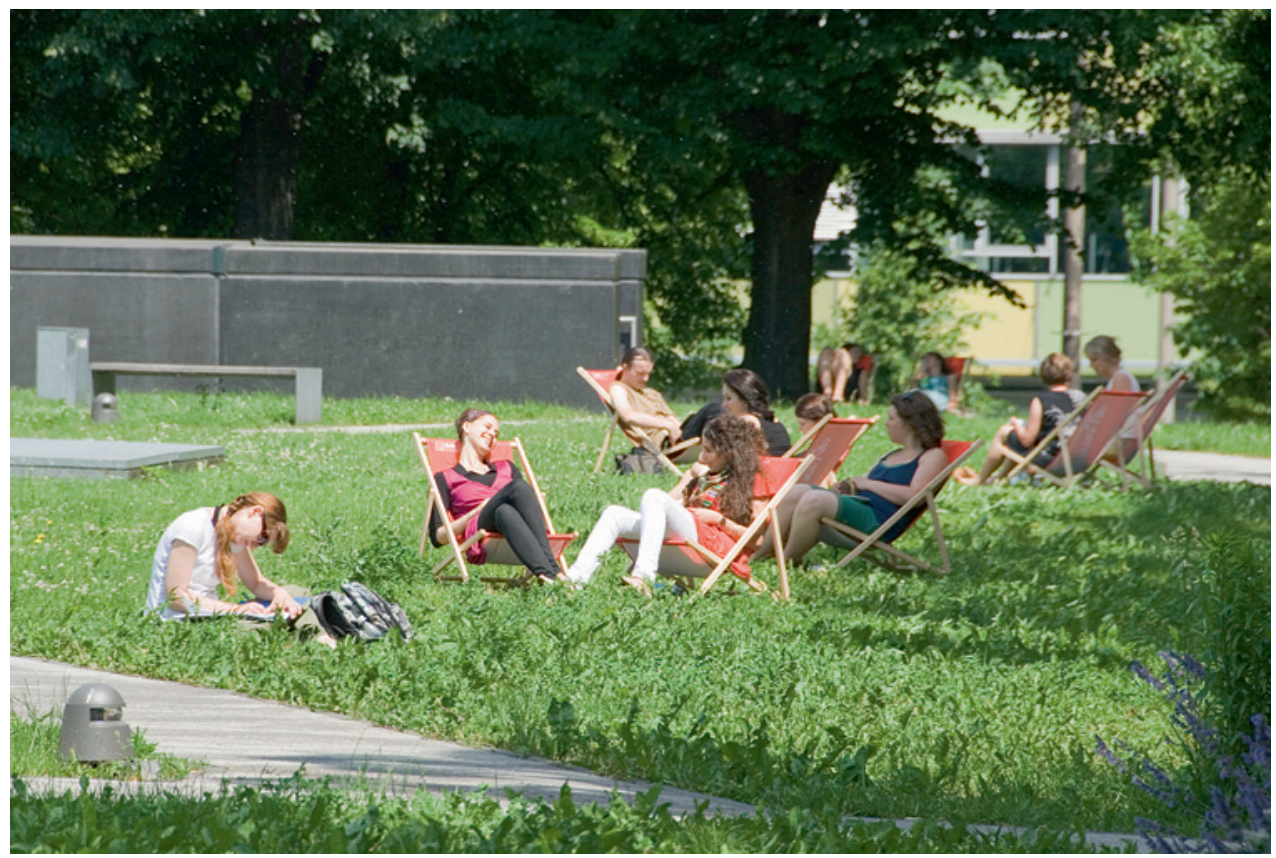

Abb. 3: „Sommerlesesaal“ auf dem Dach der SLUB (Foto: Henrik Ahlers, SLUB Dresden)

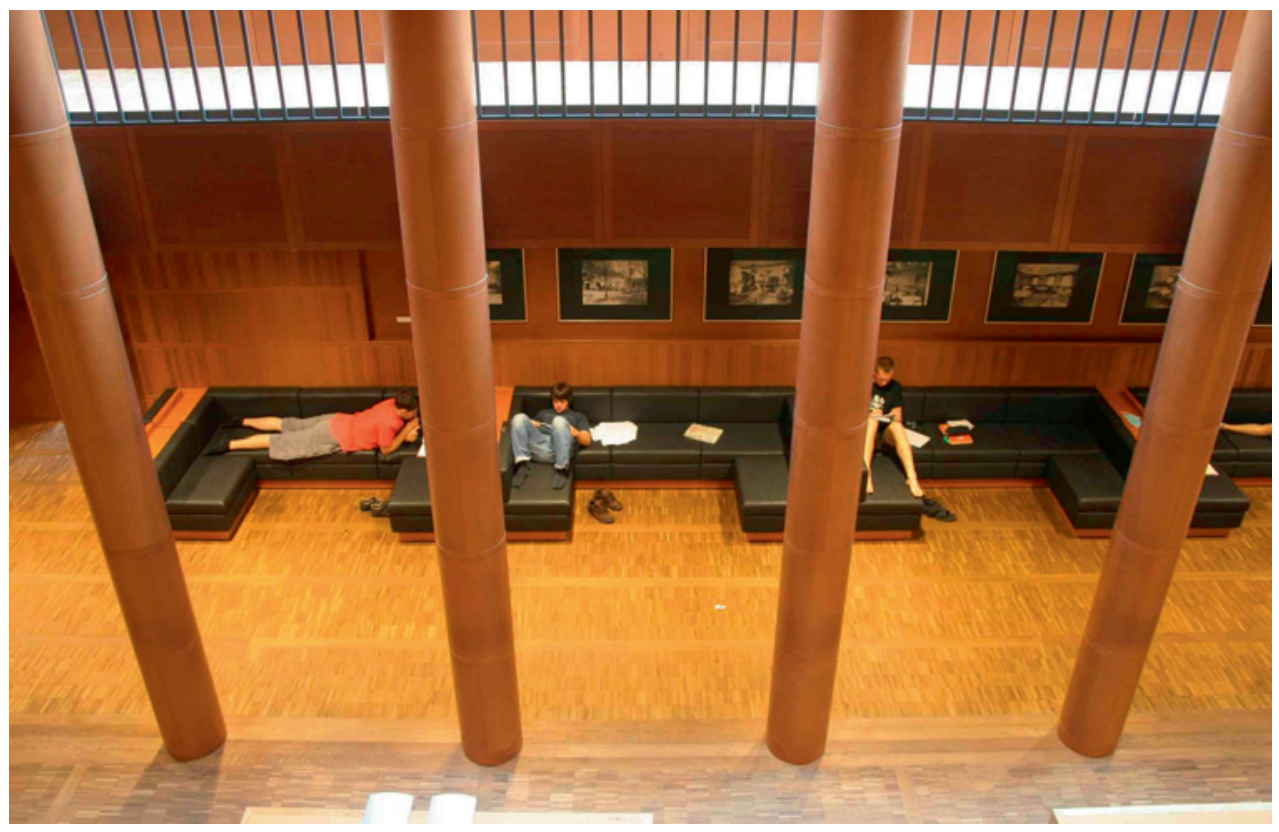

Abb. 4: Eine der Ruhezonen in der Zentralbibliothek der SLUB (Foto: Toni Klemm)

„Campuszentrale mit Wissenschaftskino, Ausstellungsflächen und Räumen für Mitmach-Aktionen". ${ }^{12}$

Die Entscheidung von Bibliotheken, in multifunktionale Räume mit Aufenthalts- und Erlebnisqualität zu investieren, bleibt dennoch richtig. Zeigen die vielfach gemeldeten Besucherrekorde doch offensichtlich eine

12 Sächsische Zeitung, 5.1.2015, Beilage „Campus“. ausgeprägte Suche nach dem Konkreten, nach materiell gefügten Gebilden, nach Körperlichkeit und scheint es bei aller Überzeugung vom Nutzen der Digitalisierung auch unter wissenschaftlicher Perspektive aus vielerlei Gründen sinnvoll, in der künftigen Wissensgesellschaft das Digitale intelligent mit dem Materiellen zu verbinden. Freilich müssen Angebotsmix und Qualität der Ausstattungsmerkmale passen, um sich mit der öffentlichrechtlichen wie privaten Konkurrenz jeweils auch tat- 
sächlich messen zu können. Ein schlichtes Sofa bildet noch keine Chill-out-Zone, ein 3-D-Drucker noch keinen Makerspace. Um der Gefahr bloßer Nachahmerei, zumal mit unzulänglichen Mitteln, zu entgehen, ist es sinnvoll, möglichst genau auf die örtlichen Rahmenbedingungen zu achten und weniger holzschnittartig als bewusst standortindividuell und feingranular zu arbeiten. Entsprechend zielt auch der eben zitierte Coworking-Space „betahaus“ in seiner Selbstbeschreibung bezeichnenderweise nicht etwa auf eine beliebige, sondern auf eine „wirklich einzigartige“ Kombination der vier integrierten Angebotswelten. Gemessen an der Internetindustrie gilt für öffentliche Bibliotheken, dass sie hinsichtlich Ressourcenumfang, Gestaltungsfreiraum und Veränderungsdynamik meist erheblich im Nachteil sind, andererseits können sie zur Leistungsentwicklung gezielt ihre ebenso vorhandenen, wesentlichen Vorteile einsetzen. Im Gegensatz zu vielen Privatunternehmen auf dem Feld von Information und Wissen genießen Bibliotheken gegenwärtig noch große gesellschaftliche Zustimmung und hohes Vertrauen. Sie gelten als weniger gewinn- denn gemeinwohlorientiert, weitgehend ideologiefrei und niedrigschwellig und können Veränderungen, die erfolgsversprechend sind, ohne fordernde Aktionäre mit ruhigerer Hand verfolgen. Wo es durch geschickte Profilierung und sorgfältige Produktbildung auf diese Weise gelingt, „Bibliothek“ neu und für die definierte Klientel jeweils „einzigartig“ zu positionieren, werden die Einrichtungen auch gegenüber kommerziellen Anbietern anhaltend konkurrenzfähig sein. Wo dagegen planloses Modisch-Sein, Zersplitterung knapper Ressourcen und unzureichende Qualität vorherrschen, könnte sich das Mantra von der Bibliothek als lebendigem Lernzentrum nur allzu rasch als Autosuggestion erweisen.

\section{Konkrete Entwicklungschancen für physische Lernråume}

Selbst bei sehr nüchterner Einschätzung bestimmter traditioneller Dienste haben Bibliotheken zu einer Zeit für die Zukunft vorzusorgen, in der die klassischen Bibliotheksaufgaben noch bei weitem nicht völlig entwertet sind. Zwar schrumpft zum Beispiel die Zahl der Entleihungen in einer Bibliothek wie der Sächsischen Landesbibliothek - Staats- und Universitätsbibliothek Dresden (SLUB) seit Anfang des Jahrzehnts jährlich um bis zu 5 Prozent, indes bleiben in absoluten Zahlen bislang immer noch rund 1,8 Millionen Vorgänge übrig. Bedenkt man zudem die teils sehr komplexen Anforderungen an ausreichend qualitätvolle neue Produkte, ist restlos klar, dass keineswegs alles, was denkbar und nützlich scheint, auch tatsächlich realisiert werden kann. Andererseits legen unsichere Zukunftsprognosen und Risiken des Scheiterns bei der Produktbildung nahe, nicht auf zu wenige Chancen zu setzen. „Ressourcen und Dienste entwickeln sich so rasant“", empfiehlt daher auch der NMC Bibliotheksreport 2014, dass Bibliotheken „mit mehreren Lösungen experimentieren, um die Bedeutung ihrer Institutionen für das neue mobile und soziale Paradigma zu erhalten." ${ }^{13}$ Den hier exemplarisch vorgestellten Bausteinen zur Konkretisierung der Bibliothek als Lernraum ist gemeinsam, dass sie unverändert am Thema speziell wissenschaftlicher Bibliotheken arbeiten. Wissen aufbauen, strukturieren und vermitteln, das bleibt auch in den neuen Realisierungen das bibliothekarische Kerngeschäft. Gegen gleichwohl durchaus denkbare Abwehrmechanismen angesichts unorthodoxer, risikobehafteter Innovationen mag ein Satz von Schopenhauer helfen. Demnach wird ein neuer Gedanke zuerst verlacht, dann bekämpft, bis er nach längerer Zeit als selbstverständlich gilt. Mit Blick auf anerkannte Pioniere aus der Technikgeschichte sowie auf die aktuellen, teils ebenso erfolgreichen wie zunächst vielleicht irritierenden Anstrengungen der Internetindustrie im Bereich von Information und Wissen sollten Bibliotheken bei ihrer Zukunftsgestaltung sicher nicht regelmäßig erst in der dritten Phase hinzustoßen.

\section{Ein Makerspace als Ort nicht-textueller Wissensproduktion und -vermittlung}

Wissensaufbau, -strukturierung und -vermittlung vollziehen sich nicht nur mittels gedruckter oder digitaler Texte, sondern in wachsendem Maße auch mit nicht-textuellen Methoden. Während die Geisteswissenschaften mit möglichst umfassend verfügbaren, gut aufbereiteten Textarchiven zufrieden sind, vermissen die Ingenieurund Naturwissenschaften in Bibliotheken bereits heute fachadäquate Lernumgebungen. Technische Sachverhalte lassen sich in visualisierter Form und am realen Objekt leichter verstehen und diskutieren. Moderne Gruppenarbeitsräume, Beamer, Whiteboard und 3-D-Simulationssoftware schaffen die dazu notwendigen Bedingungen nur teilweise. Makerspaces können dagegen einen wesentlich leistungsstärkeren Rahmen bieten.

13 NMC Horizon Report 2014 - Edition Bibliotheken, S. 20, http:// blogs.tib.eu/wp/horizon/wp-content/uploads/sites/10/2014/12/2014nmc-horizon-report-library-DE-final.pdf (8.4.2015). 


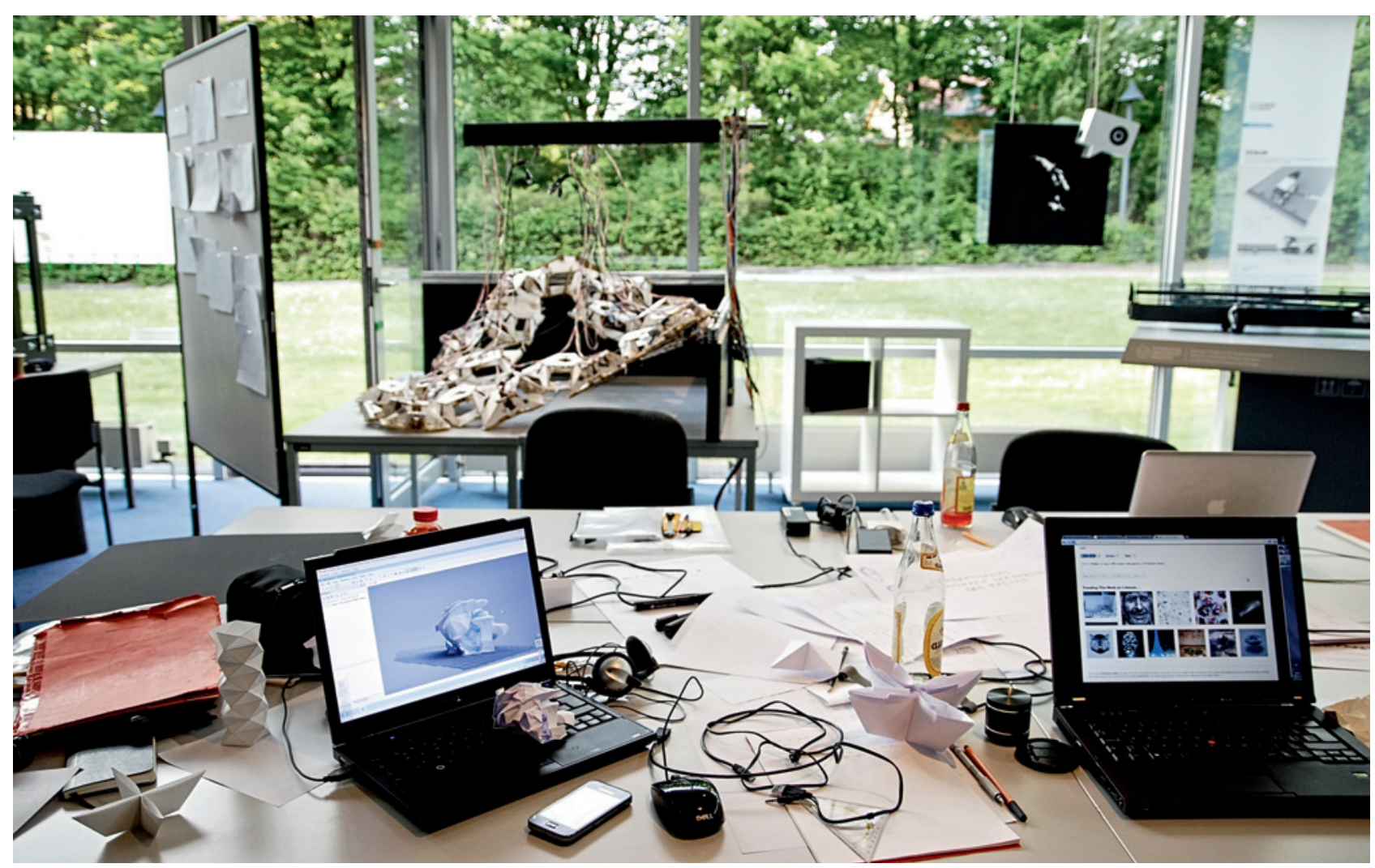

Abb. 5: SLUB Makerspace (Foto: Fanny Hauser, TU Dresden)

Makerspaces oder FabLabs sind offene High-TechWerkstätten für praktische Do-It-Yourself- bzw. Do-It-Together-Projekte. Sie dienen der Exploration neuer, wissenschaftsrelevanter Techniken, dem praktischen Erfahrungsaustausch und der Vernetzung der Fachdisziplinen. Zur Grundausstattung zählen Werkzeuge wie 3-D-Drucker, Lasercutter oder CNC-Fräsen, die moderne Produktionsverfahren zugänglich machen. Durch die persönliche Begegnung von Computerexperten, Designern und Bastlern mit jeweils spezifischem Wissen und besonderen Fähigkeiten entsteht ein interdisziplinärer Wissenspool, der neue Forschungsideen und Produkte hervorbringen kann. Wie bei Open-Access-Publikationen gilt als grundsätzliches strategisches Ziel, den offenen Zugang und die freie Weitergabe von Wissen zu fördern.

Der erste große Makerspace in deutschen wissenschaftlichen Bibliotheken entstand 2014 in der SLUB. Mit tatkräftiger Unterstützung von Wissenschaftlern der Technischen Universität Dresden sowie zweier Dresdner Fraunhofer-Institute betreibt die Bibliothek die praktische, nicht-textuelle Wissensproduktion und -vermittlung im Makerspace seitdem als regulären Angebotsstrang. Dank des regen Interesses der Dresdner Wissenschaftler und von Engagierten aus der Zivilgesellschaft ist der Makerspace der SLUB kontinuierlich mit
Workshops, Schulungen und anderen Gruppenveranstaltungen belegt. In einer gemeinsamen Summerschool diskutierten zum Beispiel Studierende der Fächer Wissensarchitektur, Technisches Design und Medieninformatik, wie sich die immer günstiger und kompakter werdenden generativen Fertigungstechniken im universitären Umfeld einsetzen lassen und schufen entsprechende Projektarbeiten. Andere Veranstaltungen betrafen etwa die Arbeit mit intelligenten Werkstoffen (Smart Materials), den gemeinsamen Eigenbau von 3-D-Druckern oder die Einführung in 3-D-Modeling-Software.

Der Makerspace der SLUB konkurriert nicht mit den häufig viel größeren Maschinenhallen der einzelnen Fachbereiche der Universität, sondern ist mit Attributen wie „Offenheit“, „Niedrigschwelligkeit“, „Interdisziplinarität“ und „Nachhaltigkeit“ ein eher propädeutischer und ergänzender, bisher fehlender Leistungsbaustein. Die Benutzungskonditionen sind weitgehend analog zum bisherigen Portfolio gestaltet. Damit entspricht eben der Gebrauch eines Plastikfilaments der Entnahme eines Lehrbuchs oder die elektronische Buchung eines bestimmten Gerätes der Vormerkung eines Mediums. Für die Bibliothek als physischen Ort bedeutet das neue Segment, dass den bislang vorhandenen, mehr oder weniger konfliktfrei koexistierenden „Bibliotheken in der Biblio- 
thek" eine weitere, vielleicht sogar besonders aussichtsreiche, hinzugefügt ist: Zur vollkommen ruhigen, auf konzentrierte Textarbeit ausgerichteten Bibliothek im Zentralen Lesesaal der SLUB und der lebendigen, kommunikativen Gruppenarbeitsbibliothek mit Rucksack und Notebook im Eingangsbereich des Hauses gesellt sich die weniger text- als anwendungsbezogene Bibliothek in einem Daniel-Düsentrieb-ähnlichen Makerspace. Als interessante Entwicklungsoptionen bleiben Integration bzw. Ausbau spezieller Beratungsangebote zu Patentinformation oder Unternehmensgründung, wie sie die Bibliothek in ihrer klassischen Form teils schon ohnehin anbietet, oder die Einrichtung einer Materialbibliothek, über die die SLUB mit verschiedenen Partnern aktuell nachdenkt. $^{14}$

\section{Die Bibliothek als zentraler Wissenschaftssalon}

Trotz vielfältiger Transfergesellschaften und Förderprogramme verläuft der Wissenstransfer aus den wissenschaftlichen Laboren in Öffentlichkeit und Wirtschaft weiter oft unbefriedigend; und wie sich in Dresden bei der Pegida-Debatte zeigte, fehlt es vielfach auch am zentralen, selbstverständlichen Ort für das kulturelle und wissenschaftliche (Streit-)Gespräch, hat der private Konversationssalon des 19. Jahrhunderts im öffentlichen Raum meist keine feste Adresse gefunden. Entsprechend wurden für Gespräche zwischen Bürgern und Landespolitikern zum Beispiel angemietete Räume im Dresdner Kongresszentrum genutzt. Aus dem vorletzten Jahrhundert der Wissenschafts- und Fortschrittsbegeisterung bis heute erhalten ist die Berliner „Urania“. Ausgehend von Initiativen Alexander von Humboldts und anderer bedeutender Wissenschaftler und mit namhaften Spenden aus der Industrie wurde die Gesellschaft 1888 eigens als Zentrum für den Dialog von Wissenschaft und Öffentlichkeit gegründet und fand in Städten wie Chemnitz, Hamburg, Jena, Wien und Zürich rasch teils bis heute aktive Nachahmer. Das Gründungskonzept umfasste neben einem volkspädagogischen Vortragsprogramm die erste Volkssternwarte der Welt sowie technische Ausstellungen mit Mitmachelementen. Heute hat der Verein „Urania Berlin“ rund 20 Mitarbeiter und über 1800 Mitglieder, darunter

14 Vgl. Burke, John J.: Makerspaces. A practical guide for librarians, Lanham 2014; Bonte, Achim u. a.: Experiment Makerspace in der SLUB. Ein neuer Ort der Wissensproduktion. In: BIS. Das Magazin der Bibliotheken in Sachsen 7,2 (2014), S. 74-76. große Firmen, die Berliner Universitäten und weitere Wissenschaftsinstitute, und organisiert jährlich über 1000 Veranstaltungen.

Falls wissenschaftliche Bibliotheken ernsthaft in diese Richtung streben, bedarf es eines Veranstaltungskalenders, der sehr weit über das bislang übliche Maß hinausweist, sowie gewidmeten Personals mit bisher in Bibliotheken teils gar nicht vorhandenen Qualifikationen. Aufwandsmindernd wirken feste Kooperationen oder gar Fusionen mit anderen öffentlichen Kultur- und Wissenschaftseinrichtungen, wie sie in einigen Großstädten schon bekannt sind. So hat etwa die Nürnberger Stadtverwaltung ihre Stadtbibliothek und die Volkshochschule 2011 organisatorisch im „Bildungscampus“ zusammengefasst oder die Stadt Potsdam das alte Gebäude der Stadt- und Landesbibliothek komplett umgebaut und 2013 als „Bildungsforum“ mit Bibliothek, Volkshochschule und „Wissenschaftsetage“ wiedereröffnet. Ein eingetragener Verein, 30 wissenschaftliche Einrichtungen und die Potsdamer Industrie- und Handelskammer organisieren in der Wissenschaftsetage Veranstaltungsformate zur Vermittlung wissenschaftlicher Inhalte. Wie auch in allen anderen Fällen wird die Entwicklung einer Bibliothek zu einem attraktiven Veranstaltungszentrum nur erfolgreich sein, wenn sie tatsächlich zu den örtlichen Rahmenbedingungen passt und - das vor allem - mit größter Konsequenz betrieben wird. Umwidmungen von Personalstellen und vorurteilsfreie Kritik des bisherigen Aufgabenportfolios sind deshalb auch hier unverzichtbar.

\section{Entwicklung einer "Smart Library“}

Nachdem uns in der ersten Phase der Digitalisierung der PC vielfältig das Leben erleichtert hat, eröffnen nun intelligente „Gegenstände“ zunehmend neue Horizonte. $\mathrm{Ob}$ Informationsaufbereitung, Heizungs- und Beleuchtungsregulierung, Diebstahlsicherung, Gerätewartung - durch immer kleinere, kaum noch merkliche Computersysteme werden Menschen wirksam unterstützt und die Kluft zwischen dinglicher und digitaler Welt verringert sich. Die Folgen des Internets der Dinge für die Organisation des Studien- und Forschungsalltags sind derzeit längst noch nicht vollständig absehbar, reichen aber zweifellos über ein effizientes Energie- und Gebäudemanagement hinaus. Mit massiven Investitionen in diesem Feld haben auch Unternehmen wie Google längst das Potential erkannt und reduzieren so zugleich ihre Abhängigkeit vom gesättigten Kerngeschäft. Der NMC Horizon Report 2014 skizziert mit einem Zeithorizont von vier bis fünf Jahren 
Anwendungsfälle für wissenschaftliche Bibliotheken. ${ }^{15}$ Wie wäre es zum Beispiel, wenn wir über an Büchern befindlichen Merkmalen jeweils unmittelbar und möglichst ausnahmslos einige komprimierte Rezensionen oder sonstige Metainformationen zum Titel vermittelt bekämen? Angesichts des nötigen Aufwands an Geld, Zeit und Expertenwissen sowie der kurzen Innovationszyklen in einem noch keineswegs gefestigten Markt ist klar, dass bei der Einrichtung solcher Dienste öffentliche Initiativen gegenüber dem Engagement einzelner Privathaushalte strategisch im Vorteil sein können.

Durch eine überdurchschnittlich transparente und zügige Medien- und Informationsbereitstellung, eine besonders enge Vernetzung mit Lehr- und Lernplattformen, die intelligente Vermittlung von Lerngruppen sowie andere praktische Lebenshilfen wie die aktuelle Anzeige von ÖPNV- und Verkehrsmeldungen, freien Mensa- oder Praktikumsplätzen lassen sich bereits heute überlegene, „smarte“ Dienste mit dem Ziel einer eindeutig profilbildenden, außergewöhnlich dichten und bequemen Servicequalität herstellen. Wenn gegenwärtig tatsächlich noch viele Bibliotheken sich hoher, ja wachsender Besucherzahlen erfreuen, könnte auch mit dieser Entwicklungsalternative besser gewährleistet werden, dass das möglichst auch in der Zukunft so bleibt. Während man für den Strategiebaustein „Wissenschaftssalon“ besonders qualifizierte Veranstaltungsmanager oder Webdesigner benötigt, erfordert die überlegene „Smart Library“ nicht zuletzt Experten für Datenschutz und Datensicherheit. Auch davon gibt es in Bibliotheken bislang definitiv zu wenig.

Mit einiger Kreativität und Unternehmergeist lassen sich den hier vorgestellten Profilschwerpunkten leicht weitere anfügen. Für manche Bibliotheken durchaus reizvoll scheint etwa auch die Idee der barocken Kunst- und Wunderkammer, wie sie als öffentliches Labor der Interdisziplinarität und Inter-Objektivität anlässlich des Wiederaufbaus des Berliner Schlosses und unter Beteiligung der Zentral- und Landesbibliothek in neuer Form realisiert werden soll. ${ }^{16}$ Aus allen Beispielen erhellt freilich, dass man sich jeweils entscheiden muss. Wohl keine Bibliothek wird in ausreichend hoher Qualität sämtlichen Entwicklungsmöglichkeiten folgen können. Es wird außerdem deutlich, dass zum Erfolg tiefgreifende Veränderungen des bibliothekarischen Berufsbilds sowie der Umbau der klassischen Stellenpläne erforderlich sind. Auf die Schlüs-

15 Vgl. NMC Horizon Report, S. 48f.

16 Vgl. Bredekamp, Horst; Eissenhauer, Michael: Keimzelle Kunstkammer, In: Das Humboldt-Forum im Berliner Schloss. Planungen, Prozesse, Perspektiven, München 2013, S. 50-57. selrolle des „Überdenkens der Funktionen und Fähigkeiten" von Bibliothekaren, verweist auch der NMC Zukunftsreport, sortiert die Herausforderung aber zumindest noch in die Kategorie der vergleichsweise verständlichen und lösbaren Aufgaben. Als schon schwieriger gilt dagegen die Bereitschaft zur notwendigen Konsequenz. „[Es] ist ein radikales Umdenken notwendig“, so die Mahnung des internationalen Expertengremiums, „um eine angemessene und nachhaltige Unterstützung für neue Initiativen und Geschäftsmodelle anbieten zu können." ${ }^{\text {"17 }}$

Bei der nicht geringen Herausforderung, einerseits in hoher Qualität laufende Verpflichtungen erfüllen zu müssen, andererseits im Interesse einer gedeihlichen $\mathrm{Zu}$ kunftsentwicklung sehr rasch wettbewerbsfähige neue Dienste zu entwickeln und Strukturen zu ändern, helfen zum Beispiel John Paul Kotters praktische Empfehlungen zum Veränderungsmanagement und sein Konzept des dualen Organisationssystems. Der immer schnellere Wandel und die wachsende Komplexität der Aufgaben kann nach Kotter von hierarchischen Organisationsmodellen nicht mehr allein bewältigt werden. Er rät deshalb zur Bildung einer agilen Netzwerkorganisation, die mit der hierarchischen Aufbauorganisation ein komplementäres System ergibt. Während sich die Aufbauorganisation mit dem Management des operativen Geschäftes beschäftigt, kümmert sich die Netzwerkorganisation um die dauerhafte Erarbeitung und Umsetzung von strategischen Initiativen und Aufgaben. Für die Netzwerkorganisation werden freiwillige Mitarbeiter herangezogen, die besonders offen für Neues sind. Sie ist in ständigem Austausch nahtlos mit dem Management des operativen Betriebs verbunden und bildet eine gleichberechtigte Ergänzung zur bestehenden hierarchischen Organisation. ${ }^{18}$ Falls dieser Dualismus tatsächlich gelingen soll, ist allgemein anzuerkennen, dass das eine so wichtig wie das andere ist. Daraus erwachsen der nötige wechselseitige Respekt und die stete Bereitschaft, sich auf die Perspektive und die Anliegen der jeweils anderen Gruppe einzulassen. Um eine übergreifende Identifikation zu ermöglichen und betriebliche Subkulturen $\mathrm{zu}$ vermeiden, bedarf es außerdem eines wirklich hohen Maßes an Transparenz der Entscheidungsprozesse wie der laufenden Arbeiten, etwa durch ein leistungsfähiges Intranet. Schließlich bleibt vorausgesetzt, dass Dimension und Permanenz der erforderlichen Veränderungen in Bibliotheken grundsätzlich akzeptiert sind.

17 Vgl. NMC Horizon Report, S. 28 und 34.

18 Vgl. Kotter, John P.: Accelerate. Building strategic agility for a faster-moving world, Harvard 2014. 
Derlei Regeln folgend, werden Bibliotheken bzw. Bibliothekare der nächsten Generation ein weniger standardisiertes Leistungs- und Raumangebot haben und damit weit weniger stereotyp als noch heute sein. Sie werden weniger bestands- als service- und erlebnisorientiert sein und im Wege kontinuierlicher, strikter Aufgabenkritik jeweils begrenzte Angebotssegmente zu echten Attraktionspunkten ausbauen. Sie werden den Informationsmarkt, ihre wichtigsten Kunden und strengsten Wettbewerber genau kennen, stets einer längerfristig tragfähigen Strategie nachzustreben versuchen und dazu jeweils sehr rasch und zielstrebig passende neue Chancen und Konstellationen nutzen. Im dicht besetzten Markt für Information und Wissen werden sich Bibliothekare der nächsten Generation prinzipiell nicht mit Mittelmaß zufrieden geben. Sie erreichen die Exzellenz - oder arbeiten weiter an ihrem Produktkatalog.

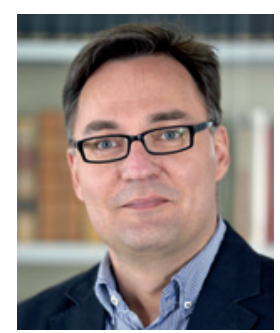

Dr. Achim Bonte SLUB Dresden 01054 Dresden achim.bonte@slub-dresden.de orcid.org/0000-0002-5642-6743 